\title{
Adsorption Geometry Determines Catalytic Selectivity in Highly Chemoselective Hydrogenation of Crotonaldehyde on $\mathrm{Ag}(111)$
}

\author{
Katrin Brandt, May E. Chiu, David J. Watson, Mintcho S. Tikhov and Richard M. Lambert
}

\section{SUPPLEMENTARY INFORMATION}

\section{TPR of Crotonaldehyde and its Hydrogenation Products}

Figure S1 shows TPR data acquired after adsorption of $~ 1 \mathrm{ML} \mathrm{H}(\mathrm{a})$ on progressively increasing amounts of crotonaldehyde (0.3 ML, 0.6 ML, $1 \mathrm{ML}$ in A-C respectively). It is clear that in every case there was a substantial and approximately constant production of crotyl alcohol, with increasing yields of butyraldehyde and, especially, butanol as the initial coverage of crotonaldehyde increased. This decreasing chemoselectivity with increasing crotonaldehyde coverage corresponds to progressive activation of the $\mathrm{C}=\mathrm{C}$ function towards hydrogenation. The amount of unreacted crotonaldehyde desorbing from the surface also increases with increasing crotonaldehyde coverage as overall conversion decreases.

The small amounts of butyraldehyde and butanol desorbing at $\sim 230 \mathrm{~K}$ and $\sim 300 \mathrm{~K}$, respectively, are possibly due to hydrogenation occurring at defect sites.
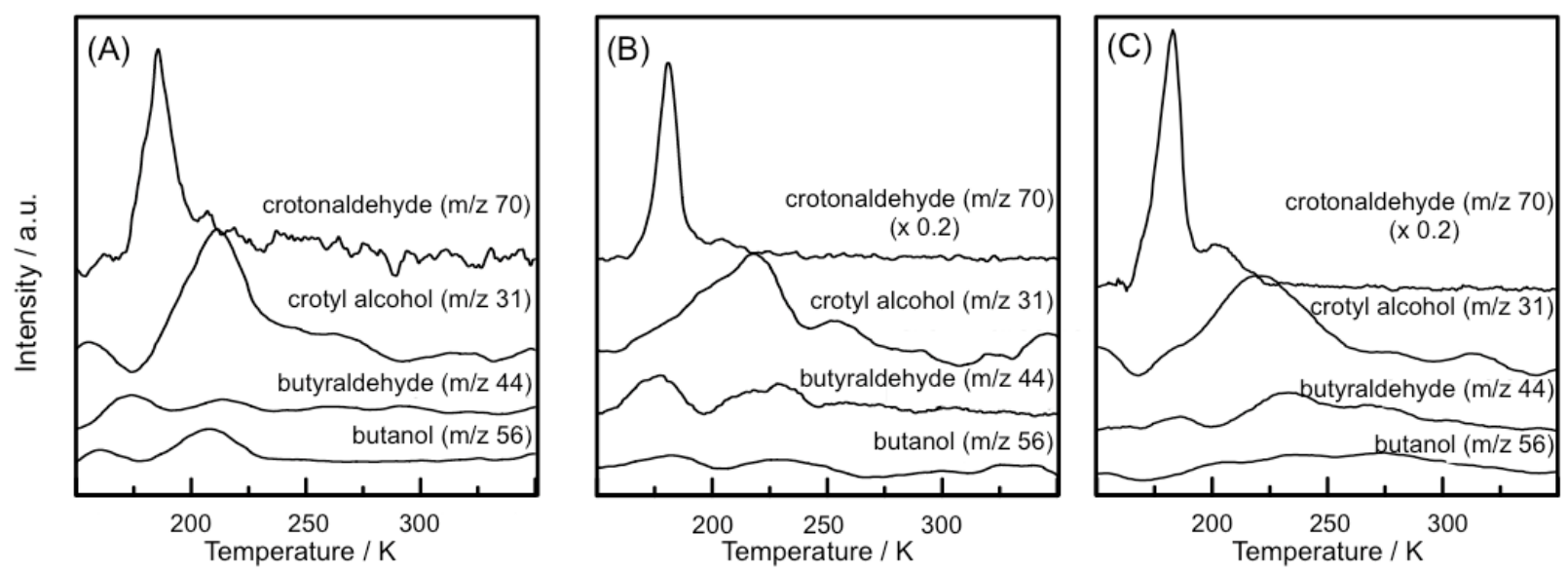

Figure S1 Dependence of reaction selectivity on crotonaldehyde coverage at fixed hydrogen coverage. TPR spectra for 0.3 ML, 0.6 ML and $1 \mathrm{ML}$ crotonaldehyde (A, B and C respectively) co-adsorbed with 1 ML H ; B-C: m/z 70 is scaled by $1 / 5$, A-C: $\mathrm{m} / \mathrm{z} 31$ is scaled by $1 / 2$. 


\section{NEXAFS of Butyraldehyde}

In contrast to the NEXAFS of crotonaldehyde and crotyl alcohol which both show a change in the orientation of the molecule with varying amounts of hydrogen, C K-edge NEXAFS of butyraldehyde reveals that the adsorption geometry is not affected by adsorbed hydrogen. NEXAFS of 0.2 ML butyraldehyde is shown in Figure S2A, where the sharp peak at $284.2 \mathrm{eV}$ is assigned to the $\mathrm{C}=\mathrm{O}$ double bond. Full analysis shows that the tilt angle with respect to the surface is $10^{\circ}$. However, after exposure to 20 minutes (2 ML) hydrogen, there was no change in the NEXAFS spectra and full analysis (Figure S2B) confirms that the bonding geometry is unchanged in the presence of hydrogen.
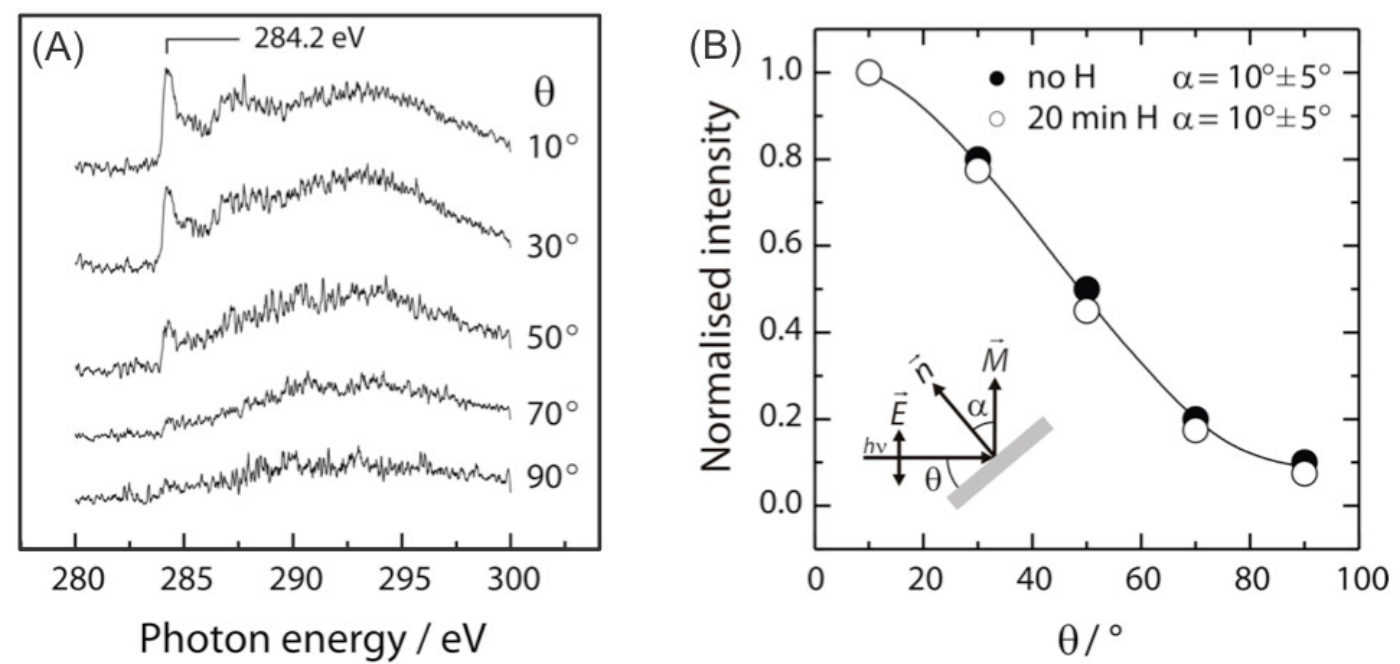

Figure S2 (A) C K-edge NEXAFS of 0.2 ML butyraldehyde, (B) Curve fitting analysis of NEXAFS intensities of 0.2 ML butyraldehyde, and the same surface after exposure to $2 \mathrm{ML}$ hydrogen. 